\title{
Ovarian mass in pregnancy: case series
}

\author{
M. R. Manvi*, Radhika Deshpande
}

Department of OBG, Kanachur Institute of Medical Sciences, Mangalore, Karnataka, India

Received: 29 June 2020

Revised: 05 September 2020

Accepted: 08 September 2020

*Correspondence:

Dr. M. R. Manvi,

E-mail: manvi.mr@gmail.com

Copyright: () the author(s), publisher and licensee Medip Academy. This is an open-access article distributed under the terms of the Creative Commons Attribution Non-Commercial License, which permits unrestricted non-commercial use, distribution, and reproduction in any medium, provided the original work is properly cited.

\begin{abstract}
Ovarian masses in pregnancy are not uncommon. Ovarian masses during pregnancy should be accurately evaluated to identify the patients who need surgical interventions from those where it can be managed conservatively. Ultrasound and magnetic resonance imaging (MRI) are safe diagnostic tools during pregnancy. The overall incidence of ovarian tumours in pregnancy is $2.4-5.7 \%$. Most masses are functional and asymptomatic and up to $70 \%$ resolve spontaneously in the second trimester. Both open surgery and laparoscopy can be performed considering the tumour diameter, gestational age and associated complications. A multidisciplinary approach is necessary in case of high suspicion of malignancy. This study evaluates the clinical features, course in pregnancy, management and pregnancy outcome in patients with ovarian masses diagnosed during pregnancy. This was a retrospective analysis of medical records of 6 patients diagnosed with ovarian mass during pregnancy in the department of Obstetrics and Gynaecology at Kanachur Institute of Medical Sciences, Mangalore from June 2019 to June 2020.
\end{abstract}

Keywords: Ovarian mass in pregnancy, Mucinous cystadenoma, Serous cystadenoma, Dermoid cyst, Paraovarian cyst

\section{INTRODUCTION}

Incidence of ovarian masses in pregnancy is becoming more common since the introduction of obstetric ultrasound in early pregnancy. The overall incidence of ovarian tumours in pregnancy is $2.4-5.7 \% .^{1}$ Of these tumours, approximately $5 \%$ are malignant. ${ }^{1}$ The prevalence of adnexal masses in pregnancy is estimated to be around $0.19-8.8 \% .^{2}$ Most cases are diagnosed in the first trimester, and the incidence appears to gradually decrease with increasing gestational age. ${ }^{2}$

Ovarian masses are characterized into benign, malignant or borderline tumours. Simple and functional cysts are very common, and they usually resolve after the first trimester. $^{3}$

The other ovarian masses in order are benign cystic teratomas, serous cystadenoma, paraovarian cyst, mucinous cystadenoma and endometrioma. Whenever malignancy is suspected in ovarian tumour during pregnancy, it is generally a germ cell tumour or borderline epithelial ovarian tumour. Mature teratomas are by far the most common persistent adnexal masses found in pregnancy.

Ovarian cysts are typically asymptomatic, but can cause pain due to rupture, bleeding or torsion. The latter requires emergency surgical intervention. During pregnancy, surgical management of ovarian cyst and complications are difficult and challenging. Second trimester is the safest period for surgical intervention during pregnancy.

Antenatally, ultrasound is considered to be the best firstline imaging to evaluate adnexal masses. ${ }^{3}$ It is known that tumour markers may be raised in pregnancy and should therefore not routinely been done. ${ }^{3}$ An alternative diagnostic tool is Magnetic resonance imaging (MRI) which is considered to be safe in pregnancy and can be helpful if the ultrasound imaging is inconclusive in evaluating whether a mass is benign or malignant. ${ }^{3}$ 
This study evaluates the clinical features, course in pregnancy, management and pregnancy outcome in patients with ovarian masses diagnosed during pregnancy.

\section{CASE SERIES}

This was a retrospective analysis of medical records of 6 patients diagnosed with ovarian mass during pregnancy in the department of Obstetrics and Gynecology at Kanachur Institute of Medical Sciences, Mangalore from June 2019 to June 2020.

This is a retrospective study of 6 patients who are diagnosed with ovarian mass during pregnancy. Out of 6 patients, 2 of them developed ovarian torsion and underwent emergency laparotomy during second trimester, patients were on regular follow up and all of them delivered by caesarean section and cystectomy was done simultaneously during caesarean section and postoperative period was uneventful.

First case was a 20 year old female, G2P1L1 at 20 weeks of gestation with history of previous Lower segment caesarean section (LSCS), anomaly scan showed single live intrauterine gestation of $20+1$ weeks with no gross anomalies evident with right ovarian cyst measuring $9.2 \times 6$ cms with peripheral mural hyperechoic specks, no septa, low level internal echoes, no significant vascularity on doppler evaluation. CA 125 - <5 U/ml. Patient had no complaints, was advised regular follow up. Growth scan at 30 weeks showed single live intrauterine gestation of 30 to 31 weeks in cephalic presentation with right ovarian cyst measuring $9.5 \times 6.2 \mathrm{cms}$ with no septa, no internal echoes, minimal margin vascularity on doppler noted. Patient was on regular follow up, scan at 35 to 36 weeks showed single live intrauterine gestation in cephalic presentation with right ovarian cyst measuring $10 \times 6 \mathrm{cms}$ with no septa, no internal echoes, minimal margin vascularity on doppler noted. Patient underwent elective LSCS at 37 weeks with right ovarian cystectomy.

Intra operative findings: Right ovarian clear cyst measuring $9 \times 10 \mathrm{cms}$ and left ovary and bilateral tubesnormal.

Post-operative period: Post-operative period was uneventful

Histopathological examination (HPE): Features suggestive of mucinous cystadenoma of right ovary.

Second case include a 24 years old female, primigravida at $20+4$ weeks presented with complaints of pain abdomen on and off since 6 months, dull aching type, non-radiating, no aggravating or relieving factors. There was no history of nausea, vomiting, fever, syncopal attack, bladder or bowel complaints. Early pregnancy scan showed single live intrauterine gestation of 6 to 7 weeks with left ovarian cyst measuring $6 \times 5.2 \mathrm{cms}$ with septations, no solid areas. CA $125-10 \mathrm{U} / \mathrm{ml}$. Patient was on regular follow up, scan at
10 weeks of gestation showed single live intrauterine gestation of 10 weeks with left ovarian cyst measuring $9 \times 5$ cms with multi septations, no solid areas, vascularity noted on cyst wall on doppler. Patient was on regular follow up, scan at 19 to 20 weeks of gestation showed single live intrauterine gestation with no obvious anomalies. Left ovary showed multi septated cyst measuring $11 \times 6 \mathrm{cms}$ with thin septations, no solid areas. Patient underwent emergency laparotomy, left ovarian cystectomy was done.

Intra operative findings: Left ovarian cyst $11 \times 10 \mathrm{cms}$, multiloculated, punctured, clear fluid drained, right tube and ovary were normal, uterus corresponding to 24 weeks size

Post-operative period: Post-operative period was uneventful

\section{Hematology of cyst fluid}

Physical examination showed quantity- $40 \mathrm{ml}$, colourblood tinged and appearance was slightly turbid.

\section{Microscopic examination}

Cell counts: Total count was 100 nucleated cells/cumm.

Cell type: Neutrophils- 60\%, Lymphocytes- $40 \%$.

\section{Background}

Numerous red blood cells (RBCs) and cyst macrophages were observed.

Patient was on regular follow up till term, scan at 36 to 37 weeks showed single live intrauterine gestation in cephalic presentation with no significant abnormality in doppler evaluation, underwent emergency LSCS in view of CPD. Post-operative period was uneventful.

Third case include a 34 years old female, G5P4L4 at 20 weeks of gestation with all previous full term normal deliveries, anomaly scan showed single live intrauterine gestation of 19 to 20 weeks with no gross anomalies evident with left adnexal cyst measuring $9 \times 6.2 \mathrm{cms}$, posterosuperior to the uterus, no septa, no solid components, no calcification, no significant internal echoes, no significant vascularity on doppler evaluation evident. No obvious features of torsion evident. CA 12518.6 U/ml. Patient was on regular follow up, scan at 37 weeks showed single live intrauterine gestation of 37 to 38 weeks in cephalic presentation with left adnexal cyst measuring $14.4 \times 8.8 \mathrm{cms}$ with no septa, low level internal echoes evident. Patient underwent emergency LSCS with left ovarian cystectomy at 37 weeks.

Intra operative findings: Left ovarian simple cyst $7 \times 7$ $\mathrm{cms}$, Right tube and ovary normal. 
Post-operative period: Post-operative period was uneventful.

HPE of the specimen: Right ovary features suggestive of para ovarian cyst.

Fourth case was a 24-year-old female, G3P2L2 at 32 weeks of gestation with history of previous 2 LSCS, scan showed single live intrauterine pregnancy of 32 weeks of gestation with left ovarian cyst measuring $8.8 \times 6.4 \times 4.1$ cms with few septations, maximum thickness of septation measures $2.8 \mathrm{~mm}$ with no obvious solid nodularity. CA 125-8 U/ml. Patient was on regular follow up, at $34+2$ weeks of gestation, patient presented with c/o pain abdomen since 1-day, dull aching type, non-radiating, no aggravating or relieving factors. There was no history of nausea, vomiting, fever, scan showed single live intrauterine gestation of 34 weeks with cyst in the left side of abdomen, measuring $9 \times 4.4 \mathrm{~cm}$, septa noted, minimal peripheral local vascularity on Doppler evaluation notedovarian cyst with? Partial torsion. Patient underwent emergency LSCS with left ovarian cystectomy.

Intra operative findings: Left ovarian cyst $7 \times 7 \mathrm{~cm}$ with torsion, right tube and ovary- normal

Post-operative period: Post-operative period was uneventful.

HPE of specimen: Features suggestive of mucinous cystadenoma of left ovary.

Fifth case include a 27 year old female, primigravida at $39+2$ weeks of gestation presented with the complains of labour pain since 1 day, on examination, patient was in active labour, cervix was $75 \%$ effaced, $3-4 \mathrm{~cm}$ dilated, bag of membranes were forming, vertex was at -2 station, artificial rupture of membranes done, clear liquor drained around 15-20 ml, labour augmented with 5 units of oxytocin drip, contractions were monitored, patient was getting adequate contractions, fetal heart sound was monitored, in spite of getting adequate contractions, labour did not progress well, patient was taken up for emergency LSCS in view of non-progress of labour due to CPD.

Intra operative findings: Left ovarian cyst measuring $5 \times 4$ cms, cystectomy done, right tube and ovary- normal.

Post-operative period: Post-operative period was uneventful.

HPE of specimen: Serous cystadenoma of left ovary.

Patient had regular Ante natal check-ups (ANCs) elsewhere. Scan at 6 weeks showed single live intrauterine gestation corresponding to 6 weeks and 3 days. Nuchal translucency (NT) scan showed single live intrauterine gestation at 13 weeks and 1 day with NT of $1.1 \mathrm{~mm}$, nasal bone visualised. Anomaly scan showed single live intrauterine gestation at 20 weeks and 2 days with no obvious anomalies evident. Growth scan showed single live intrauterine gestation at $31+5$ weeks in cephalic presentation, with adequate liquor, anterior placenta, Estimated fetal weight (EFW) of $1806 \pm 126 \mathrm{gm}$.

Sixth case include a 30 years old female, primigravida at 11 weeks of gestation scan showed $9.7 \times 6 \mathrm{cms}$ mixed echogenic lobulated focus in the left adnexa. Medial hyperechogenecity about $5.5 \mathrm{cms}$ with shadowing noted. Lateral cystic component $(4.2 \times 3.5 \mathrm{cms})$ noted. No significant vascularity on doppler evaluation evident. No right adnexal lesion evident. No free fluid evident.

Impression: Mixed echogenic focus at left adnexa? Ovarian dermoid.

CA $125-7 \mathrm{U} / \mathrm{ml}$. Scan at $12+4$ weeks showed single live intrauterine gestation with echogenic lesion in the left adnexa possibly left ovarian dermoid. Left adnexa shows echogenic lesion measuring around $52 \times 50 \mathrm{~mm}$ on the lateral side of echogenic lesion, cystic lesions noted measuring 23 and $18 \mathrm{~mm}$. No significant vascularity on doppler study. NT- $1.8 \mathrm{~mm}$. Patient underwent emergency laparotomy as she developed pain abdomen at 18 weeks, pain was dull aching type, non-radiating, no aggravating or relieving factors. There was no history of nausea, vomiting, fever. Left ovarian cystectomy was done.

Post-operative period: Post-operative period was uneventful.

HPE of specimen: Features suggestive of left ovarian dermoid.

Patient was on regular follow up, anomaly scan showed single live intrauterine gestation of 21 to 22 weeks with no gross anomalies evident. Growth scan at 34 weeks showed single live intrauterine gestation in cephalic presentation, Amniotic fluid index (AFI)- adequate, placenta- fundal posterior, EFW- $2298 \pm 345 \mathrm{gm}$.

At 37 weeks of gestation, patient underwent elective LSCS in view of precious pregnancy. Post-operative period was uneventful.

\section{DISCUSSION}

Frequency of ovarian tumours being coexistent with pregnancy is 1:1000 and among these frequencies of being malignant is approximately $1: 15000$ to $1: 32000$ pregnancies. ${ }^{4}$ Most common ovarian masses during pregnancy are functional cysts of ovary (corpus luteum of pregnancy and theca-lutein cysts). The other ovarian masses in order are benign cystic teratomas, serous cyst adenomas, para-ovarian cysts, mucinous cystadenomas and endometriomas. ${ }^{4}$

The mucinous cystadenomas are one of the benign epithelial ovarian tumours which are $>5 \mathrm{~cm}$ in size, unilateral and multilocular with smooth surface and 
contain mucinous fluid. They comprise $12-15 \%$ of all ovarian tumors. Around $75 \%$ of all mucinous tumors are benign, while $10 \%$ are borderline and $15 \%$ are invasive carcinomas. The benign mucinous tumors are most common in the third to fifth decades of life and may be 20$30 \mathrm{~cm}$ in size. ${ }^{5}$ Giant cysts are found in less than $1 \%$ of the cases of ovarian cysts with pregnancy. ${ }^{6}$

Torsion is the most common and serious complication of benign ovarian cysts during pregnancy, occurs in 10 to $20 \%$ of cases. ${ }^{7-9}$ Ovarian torsions occur most commonly during first trimester, less commonly during second trimester and rarely during third trimester. It is defined as complete or partial rotation of the ovarian vascular pedicle on its long axis, usually involving the tube and ovary. Different factors have been associated with an increased risk of adnexal torsion in pregnancy, including gestational age (58\% of cases in the first trimester), mass size (greater risk for masses with diameters between 6 and $10 \mathrm{~cm}$, and ovarian stimulation ( $73 \%$ of cases associated with the use of assisted reproductive technology). ${ }^{2}$ Clinical presentation includes abdominal pain, nausea, vomiting, and eventually fever and leucocytosis. ${ }^{2}$ Diagnosis under imaging shows larger and more rounded ovary, heterogeneous stromal echogenicity, multiple small size follicles in periphery, decreased intraparenchymal blood flow, and the "Whirlpool sign". Surgery is the plan of management. The risk of rupture $(0-5 \%)$ and other mechanical complications such as haemorrhage and obstruction $(9 \%)$ is less frequently described. ${ }^{2,10,11}$ The other complications which might occur are infection, malignancy, impaction of cyst in pelvis, obstructed labour and malpresentations of fetus. ${ }^{4}$

Para-ovarian cysts are remnants of wolffian duct in the mesosalpinx that do not arise from the ovary. These are responsible for about $10-20 \%$ of all adnexal masses. They arise from broad ligament, usually from mesothelial and paramesonephric elements or rarely from mesonephric remnants. These cysts may be complicated by haemorrhage, torsion and rupture. The sonographic diagnosis can be made by demonstration of a normal ipsilateral ovary close to but separate from the adnexal cyst, usually thin walled, unilocular simple cyst.

Dermoid cyst constitutes 20 to $40 \%$ of ovarian tumours in pregnancy. It is the most common non-functional ovarian masses in premenopausal women. Accounts for 15 to $20 \%$ of ovarian tumours, bilateral in 15 to $20 \%$. Torsion is the most common complication, during pregnancy, risk is reported to be approximately 3 to $15 \%$. On cut section, content is predominantly sebaceous material with hair follicle, may also contain teeth, bone, thyroid, cartilage and bronchial mucous membrane on the wall.

While evaluating a dermoid cyst by ultrasound, characteristic features are:

- Rokitansky nodule, a hyperechoic nodule with acoustic shadowing in a background of low-level echoes.
- 'Tip of the iceberg' phenomenon, where a highly echogenic cyst, contents of sebum and hair, causes posterior attenuation of sound.

- 'Dermoid mesh', multiple interdigitating lines and dots which are seen when hair is floating in sebum. ${ }^{12}$

Dermoid cysts are benign tumours, can give rise to symptoms due to torsion.

The serous cystadenomas are one of the benign epithelial ovarian tumours which are large simple cyst $>5 \mathrm{~cm}$, bilateral in $20 \%$ of cases, with thin septations, may be multilocular, smooth surface and contain clear serous fluid. They comprise $50 \%$ of all ovarian tumors, 5 to $10 \%$ have borderline malignant potential and 20 to $25 \%$ are malignant. It is common in third to fifth decades of life. In $15 \%$ of cases, papillary growths projects outwards perforating the cyst wall. Microscopically, tiny spherical laminated structures found in areas of cellular degeneration called Psammoma bodies are found in $15 \%$ of cases. Surgical intervention during pregnancy is indicated for large and symptomatic tumours and those that appear highly suspicious for malignancy on imaging or laboratory tests.

\section{Management}

Management of ovarian cysts depends on the size of cysts. Most of the cysts which measure less than $6 \mathrm{cms}$ and which have benign looking features on ultrasonography (USG) can be managed conservatively and regular follow up is done as most of them resolve spontaneously over time. Cysts which measure more than $10 \mathrm{~cm}$ are generally removed due to risk of complications like torsion, rupture and increase chances of malignancy. Management of cysts with diameter between $6-10 \mathrm{~cm}$ is controversial, if ultrasound features show solid components, papillary excrescences, septae and nodules, it is better to remove because of the risk of malignancy.

If features are suggestive of benign cyst, it is followed up by serial ultrasonography. Benign cysts may necessitate emergency laparotomy and exploration if complications like torsion, rupture or necrosis arise as seen in as many as $50 \%$ cases. $^{2}$ Now a days, due to advent of modern techniques such as MRI, transvaginal colour doppler, high resolution ultrasound, conservative management has become quite easy.

Management of ovarian cysts during pregnancy should be done according to period of gestation. Most common ovarian cysts encountered during pregnancy are corpus luteal cysts. They usually resolve by 12-16 weeks, followed up regularly. By 16 weeks, implantation of pregnancy is more secure, chances of abortion is less. Persisting ovarian cysts beyond 16 weeks of gestation are managed by simple cystectomy or ovariotomy as indicated till 28 weeks. Beyond this gestation, there is risk of preterm labour if surgery is considered. Emergency laparotomy is considered in any gestational age whenever 
complications arise such as torsion, rupture, haemorrhage, necrosis or features suggestive of malignancy. ${ }^{2}$

\section{CONCLUSION}

Most adnexal masses, which arise in pregnancy, are functional and asymptomatic and resolve spontaneously, invasive cancer is rare in pregnancy. Persistent adnexal masses carry a low risk of malignancy, and ultrasonography is the preferred and safest method to assess this risk. In asymptomatic pregnant women without predictors of complications, expectant management is generally the most suitable option. If strong clinical suspicion of malignancy or presence of acute abdomen or severe clinical manifestations occur, surgical treatment is indicated by laparotomy or laparoscopy. Complication of surgical management of ovarian mass is uncommon in second trimester of pregnancy.

Funding: No funding sources Conflict of interest: None declared

Ethical approval: Not required

\section{REFERENCES}

1. Kwon YS, Mok JE, Lim KT, Lee IH, Kim TJ, Lee KH et al. Ovarian Cancer during Pregnancy: Clinical and Pregnancy Outcome. J Korean Med Sci. 2010;25:2304.

2. JoãoCavaco-Gomes, Moreira CJ, Rocha A, Mota R, Paiva V, Costa A. Investigation and Management of Adnexal Masses in Pregnancy. Hindawi Publishing Corporation Scientifica. 2016;3012802:9.

3. Eichenberger-Gautschi T, Smith A and Sayasneh A. Ovarian masses in pregnancy: a single centre retrospective study. BJMP. 2018;11(1):a1109.
4. Dahiya P, Latika. Ovarian mass in pregnancy: a case report. Int J Reprod Contracept Obstet Gynecol. 2015;4:915-7.

5. Ioffe OB, Simsir A, Silverberg SG. Pathology. In: Practical Gynecologic Oncology. 3rd ed. Philadelphia. Berek JS, Hacker NF, editors. Lippincott Williams \& Wilkins Company. 2000:213214.

6. Aujang RE. Giant ovarian cyst and pregnancy. Case report and literature review. Ginecol Obstet Mex. 2011;79:235-8.

7. White $M$ and Stella J. Ovarian torsion: 10-year perspective. Emergency Medicine Australasia. 2005;17(3):231-7.

8. Houry D and Abbott JT. Ovarian torsion: a fifteenyear review. Annals of Emergency Medicine. 2001;38(2):156-9.

9. Tsafrir Z, Hasson J, Levin I, Solomon E, Lessing JB, Azem F. Adnexal torsion: cystectomy and ovarian fixation are equally important in preventing recurrence. European Journal of Obstetrics Gynecology and Reproductive Biology. 2012;162(2)203-5.

10. Kumari I, Kaur S, Mohan H, Huria A. Adnexal masses in pregnancy: a 5-year review. The Australian and New Zealand Journal of Obstetrics and Gynaecology. 2006;46(1):52-4.

11. Koo Y, Kim HJ, Limetal K. Laparotomy versus laparoscopy for the treatment of adnexal masses during pregnancy. Australian and New Zealand Journal of Obstetrics and Gynaecology. 2012;52(1):34-8.

12. de Haan J, VerHeecke M, Mant FA. Management of ovarian cysts and cancer in pregnancy. Facts Views Vis Obgyn. 2015;7(1):25-31.

Cite this article as: Manvi MR, Deshpande R. Ovarian mass in pregnancy: case series. Int J Reprod Contracept Obstet Gynecol 2020;9:42904. 